\title{
Article \\ Role of Standardized Plant Extracts in Controlling Alcohol Withdrawal Syndrome-An Experimental Study
}

\author{
Ijasul M. Haque ${ }^{1}{ }^{\mathbb{D}}$, Akhilesh Mishra ${ }^{2}$, Bhupinder Singh Kalra ${ }^{1}{ }^{\mathbb{C}}$ and Shalini Chawla ${ }^{1, *}$ \\ 1 Department of Pharmacology, Maulana Azad Medical College, New Delhi 110002, India; \\ ijasulhaqmm@gmail.com (I.M.H.); drbskalra@gmail.com (B.S.K.) \\ 2 Central Animal Facility, Maulana Azad Medical College, New Delhi 110002, India; drakhileshvet@gmail.com \\ * Correspondence: drshalinichawla@gmail.com
}

Citation: Haque, I.M.; Mishra, A.;

Kalra, B.S.; Chawla, S. Role of

Standardized Plant Extracts in Controlling Alcohol Withdrawal Syndrome-An Experimental Study. Brain Sci. 2021, 11, 919. https:// doi.org/10.3390/brainsci11070919

Academic Editor: Chitra Mandyam

Received: 15 June 2021

Accepted: 7 July 2021

Published: 12 July 2021

Publisher's Note: MDPI stays neutral with regard to jurisdictional claims in published maps and institutional affiliations.

\begin{abstract}
Patients with alcohol use disorder experience alcohol withdrawal syndrome due to the sudden cessation of alcohol. This study was designed to evaluate the protective effect of Ashwagandha and Brahmi on alcohol withdrawal in rats. Thirty rats of either sex were taken and randomly divided into 6 groups $(n=5)$. Their normal diet was replaced by a modified liquid diet (MLD). Ethanol was added gradually except in the MLD group for a period of 21 days and withdrawn suddenly. Four treatment groups were administered Ashwagandha ( $3.75 \mathrm{mg}$ of withanolide glycosides per $\mathrm{kg}$ body weight), Brahmi (10 mg of bacosides per $\mathrm{kg}$ body weight), Ashwagandha + Brahmi (3.75 mg withanolide glycosides $+10 \mathrm{mg}$ bacosides per $\mathrm{kg}$ body weight) orally and diazepam ( $1 \mathrm{mg} / \mathrm{kg}$ body weight, i.p.) $45 \mathrm{~min}$ before alcohol withdrawal. Rats were assessed for behavioural changes (agitation score and stereotypic behaviour), anxiety and locomotor activity at 2nd and 6th hours of alcohol withdrawal. Pentylenetetrazol (PTZ) kindling seizures were assessed at 6th hour of alcohol withdrawal. Ashwagandha and Brahmi alone and in combination significantly reduced the behavioural changes in alcohol withdrawal rats at 2 nd hour and their combination in 6th hour. Ashwagandha and Brahmi suppressed PTZ kindling seizures effectively and improved locomotory activity at 2nd hour and 6th hour of alcohol withdrawal. Reduction in anxiety was significant among Ashwagandha at 2nd hour and the combination group at 2 nd and 6th hour. The results were comparable to diazepam. Ashwagandha and Brahmi have beneficial effects in controlling the behavioural changes, anxiety and seizures in alcohol withdrawal symptoms in rats and improved locomotory activity.
\end{abstract}

Keywords: Ashwagandha; Brahmi; alcohol withdrawal syndrome; seizures; diazepam

\section{Introduction}

Alcohol (ethanol or ethyl alcohol) is a commonly used psychoactive substance with dependence-producing properties. Globally, alcohol use disorder (AUD) is a major health problem and is associated with social, mental, physical and legal consequences. It is generally associated with cravings, tolerance and withdrawal symptoms upon cessation [1]. Alcohol withdrawal syndrome (AWS) is a well-known condition occurring after intentional or unintentional abrupt cessation of heavy or chronic drinking and it occurs in about $8 \%$ of hospitalized AUD inpatients [2]. AWS is characterized by tremors, anxiety, sweating, nausea and tachycardia and in severe cases, may involve hallucinations, seizures and delirium tremens. Untreated, AWS can be fatal [1].

Alcohol is a central nervous system depressant that produces euphoria and behavioural excitation at low blood concentrations due to increased glutamate binding to N-methyl-D-aspartate (NMDA) receptors; at higher concentrations, it leads to acute intoxication by potentiation of the GABA effects [3]. Prolonged alcohol use leads to the development of tolerance and physical dependence, which may result from compensatory functional changes by downregulation of GABA receptors and increased expression of NMDA receptors. Abrupt cessation of chronic alcohol consumption unmasks these changes 
with a glutamate-mediated CNS excitation resulting in autonomic overactivity and neuropsychiatric complications such as delirium and seizures [4]. Thus, patients experiencing alcohol withdrawal have decreased GABA-ergic functioning and increased glutamatergic action throughout the central nervous system.

Management for any patient with suspected alcohol withdrawal is initial resuscitation and rehydration. Benzodiazepines (BZD) are the first line pharmacological agent for treating AWS. The benzodiazepines most used for this purpose are lorazepam, chlordiazepoxide, oxazepam and diazepam. They act as central GABA-A agonists, increasing the frequency of GABA-receptor channel opening leading to more influx of chloride ions and providing an inhibitory effect which is similar to that of ethanol [5]. BZDs are associated with potentially life-threatening side effects, including the risk of dependency and withdrawal symptoms, increased risks of falls and respiratory failure. In addition, psychological side effects, cognitive impairments, depressive mood and sleep disorders have been described, as well as increased impulsivity that could lead to suicidal behaviours [6]. In view of the available literature, there is a need for better and safer pharmacological agents for managing AWS.

Withania somnifera, commonly known as Ashwagandha, has been used for centuries in Ayurvedic medicine to increase longevity and vitality [7]. Various preclinical studies have shown that Ashwagandha has anticonvulsant [8], antidepressant [9], antianxiety [10], hepatoprotective [11] effects, etc. Ashwagandha preparations have been found to have a potential therapeutic role in almost every CNS-related disorders. Glycowithanolides withaferin- A and sitoindosides VII-X isolated from Ashwagandha significantly reversed ibotenic acid-induced cognitive defects in Alzheimer's disease model [12]. Another study demonstrated that chronic oral administration of withanoside attenuated the axonal, dendritic and synaptic losses and memory deficits induced by amyloid peptide A $\beta$ (25-35) in mice [13]. It can modulate GABAergic, cholinergic and oxidative systems in CNS [14].

Bacopa monnieri, commonly known as Brahmi is a nootropic plant in Ayurveda that has been studied widely for its cognition-enhancing, antidepressant, antihypertensive, antiasthmatic, antiulcer, analgesic, neuroprotective, hepatoprotective and nephroprotective properties. Bacoside-A is the major chemical entity responsible for Brahmi's well-known nootropic effect as well as other neuromodulatory, hepatoprotective and, antioxidant activities [15]. Few preclinical studies have shown that Brahmi treatment potentiates a therapeutic effect by reversing the alterations in $\mathrm{GABA}$ and $\mathrm{GABA}_{\mathrm{A}}$ receptor binding that occur during epilepsy, resulting an increased GABA mediated inhibition in the overstimulated cerebral cortex neurons $[15,16]$. Another study showed improvement in spatial learning performance and enhanced memory retention in rats treated with Brahmi extract clearly indicating that exposure to Brahmi improved learning and memory [17]. Brahmi also showed strong and dose-dependent antidepressant effects in different mice models [18]. Another study in epileptic rat model concluded that Brahmi potentiates a therapeutic effect by reversing the alterations in general GABA, GABAA, GABAB receptor binding, GABAA receptor subunits, GAD and $C R E B$ gene expression that occurs during epilepsy, resulting an increased GABA mediated inhibition in the over stimulated cerebral cortex neurons [16].

In preclinical studies, animals exhibit alcohol abstinence symptoms like those observed in humans. The anxiety and depressive-like behaviour in rodent models mimics human dysphoric emotion related to alcohol abstinence and may aid in testing the therapeutic potential of promising agents for alcoholism [19]. In most of the studies, modified liquid diet model of chronic ethanol administration was used. A study was done to validate the modified liquid diet model of chronic ethanol administration. They concluded that it may be used as a valid means to investigate ethanol abuse and dependence in an experimental context and can be used to assess the pharmacological profile of drugs on alcohol withdrawal syndrome in rats [20].

Ashwagandha and Brahmi with their GABA agonist activity may have the potential to control alcohol withdrawal symptoms. The objective of the study was to study and compare the effect of Withania somnifera (Ashwagandha) and Bacopa monnieri (Brahmi) 
individually and their combination with Diazepam on alcohol withdrawal syndrome in rats.

\section{Materials and Methods}

\subsection{Materials and Reagents}

Ashwagandha (Withania somnifera) extract containing 35\% withanolide glycosides and Brahmi (Bacopa monnieri) extract containing 21.4\% total bacoside were supplied by Arjuna Natural Pvt. Ltd., Aluva, India. Diazepam and ethanol were purchased and used in the study. Reference standards of Withaferin A (\% purity $\geq 99.0)$, Withanoside IV (\% purity $\geq 99.0$ ) and Withanoside $\mathrm{V}$ (\% purity $\geq 99.0)$ were purchased from Chromadex (Los Angeles, CA, USA) and Bacoside A3 (\% purity $\geq 99.0$ ) were purchased from Sigma. Acetonitrile, methanol and water (LCMS grade) were purchased from Merck (Mumbai, India). The Waters ${ }^{\circledR}$ ACQUITY UPLC (Milford, MA, USA) with a photo-diode array (PDA) detector and Triple Quadrupole (Waters Quattro Premier XE, Poway, CA, USA) mass spectrometer with an electrospray ionization (ESI) source was used for the mass spectrometric analysis.

\subsection{LCMS Analysis Method of Ashwagandha Extract}

A total of $0.1 \mathrm{mg} / \mathrm{mL}$ of reference standards Withaferin A, Withanoside IV and Withanoside $\mathrm{V}$ diluted in $25 \mathrm{~mL}$ methanol (LCMS grade) were prepared. A total of $10 \mathrm{mg}$ of Ashwagandha extract was dissolved in methanol (LCMS grade) and made up to $25 \mathrm{~mL}$ volumetric flask. The sample was clarified using Millipore filters $(0.22 \mu \mathrm{m})$, degassed for one minute and subjected to LCMS analysis. Analytes were quantified using MRM method. Withanoside IV and Withanoside V were ionized in the negative mode and Withaferin A was ionized in the positive mode. All the MS parameters were manually fine-tuned to obtain the highest MRM signals. The following MRM transitions of precursor ions to product ions were used: $\mathrm{m} / \mathrm{z} 827.441 \rightarrow 763.359$ for Withanoside IV; $\mathrm{m} / \mathrm{z} 811.063 \rightarrow 765.51$ for Withanoside V; and $\mathrm{m} / \mathrm{z} 471.171 \rightarrow 94.95$ for Withaferin A. The compounds were separated on a C18 Kinetex column $(50 \mathrm{~mm} \times 2.1 \mathrm{~mm} \times 1.7 \mu \mathrm{m})$ at a column temperature of $35^{\circ} \mathrm{C}$. The mobile phase was composed of water (A) and acetonitrile (B) with a gradient elution program as per Aboli Girme et al. The chromatograms were acquired at a flow rate of $0.3 \mathrm{~mL} / \mathrm{min}$ with an injection volume of $5 \mu \mathrm{L}$ [21]. ESI-MS spectra of Ashwagandha extract showing Withanoside IV at Rt $7.44 \mathrm{~min} \mathrm{m/z} 827.47$ formate adduct, Withaferin A at Rt $9.09 \mathrm{~min} \mathrm{~m} / \mathrm{z} 471.29(\mathrm{M}+\mathrm{H})^{+}$and Withanoside $\mathrm{V}$ at Rt $9.13 \mathrm{~min} \mathrm{~m} / \mathrm{z} 811.38$ formate adduct were represented in Figure 1.

\subsection{LCMS Analysis Method of Bacopa Extract}

A total of $0.1 \mathrm{mg} / \mathrm{mL}$ of the reference standard of Bacoside A3 diluted in $25 \mathrm{~mL}$ methanol (LCMS grade) was prepared. A total of $25 \mathrm{mg}$ of Bacopa extract was dissolved in methanol (LCMS grade) and was made up to $50 \mathrm{~mL}$ volumetric flask. The sample was filtered using Millipore filters $(0.22 \mu \mathrm{m})$ for one minute and subjected to LCMS analysis. An aliquot of $10 \mu \mathrm{L}$ of the sample solution was used for LC-MS analysis with a total run time of $16 \mathrm{~min}$. Mass spectra were recorded in the positive ionization mode and with the full scan (m/z 100-1000). The compounds were separated on a C18 Kinetex column $(50 \mathrm{~mm} \times 2.1 \mathrm{~mm} \times 1.7 \mu \mathrm{m})$ with a column temperature of $45^{\circ} \mathrm{C}$. The mobile phase was composed of $0.1 \%$ formic acid in water (A) and $0.1 \%$ formic acid in acetonitrile (B) with a gradient elution program. The chromatograms were acquired at a flow rate of $0.3 \mathrm{~mL} / \mathrm{min}$. All the acquisition and analysis of the data were controlled by Mass Lynx Data Acquisition Software version 4.1. TIC of the samples exhibited several peaks along with their retention time (Rt) indicating the presence of bacosides in the Bacopa extract. The jujubogenin glycosides (Bacoside A3 and Bacopaside $\mathrm{X}$ ) showed the characteristic $\mathrm{m} / \mathrm{z}$ 455 [aglycone $\left.+\mathrm{H}-\mathrm{H}_{2} \mathrm{O}\right]^{+}$, while pseudojujubogenin glycosides (Bacopaside I, Bacopaside II and Bacopasaponin C) showed the main fragment ion at $\mathrm{m} / \mathrm{z} 473$ [aglycone $+\mathrm{H}]^{+}$. Some of the important possible bacosides were identified with the help of the ESI-MS spectral 
analysis, which were already reported [22]. The possible bacosides identified in sample were Bacoside A3 at Rt of $12.66 \mathrm{~min}, \mathrm{~m} / \mathrm{z}$ 929. A total of $41[\mathrm{M}+\mathrm{H}]^{+}$, Bacopaside II at Rt of $12.92 \mathrm{~min}, \mathrm{~m} / \mathrm{z} 929.52[\mathrm{M}+\mathrm{H}]^{+}$, Bacopaside $\mathrm{X}$ at Rt of $13.36 \mathrm{~min}, \mathrm{~m} / \mathrm{z} 899.67[\mathrm{M}+\mathrm{H}]^{+}$, Bacopasaponin C at Rt of $13.57 \mathrm{~min}, \mathrm{~m} / \mathrm{z} 899.42[\mathrm{M}+\mathrm{H}]^{+}$, Bacopaside I at Rt of $13.72 \mathrm{~min}$, $\mathrm{m} / \mathrm{z} 979.25[\mathrm{M}+\mathrm{H}]^{+}$and Bacoside $\mathrm{N} 1$ at Rt of $14.07 \mathrm{~min} \mathrm{~m} / \mathrm{z} 767.41[\mathrm{M}+\mathrm{H}]^{+}$(Figure 2).

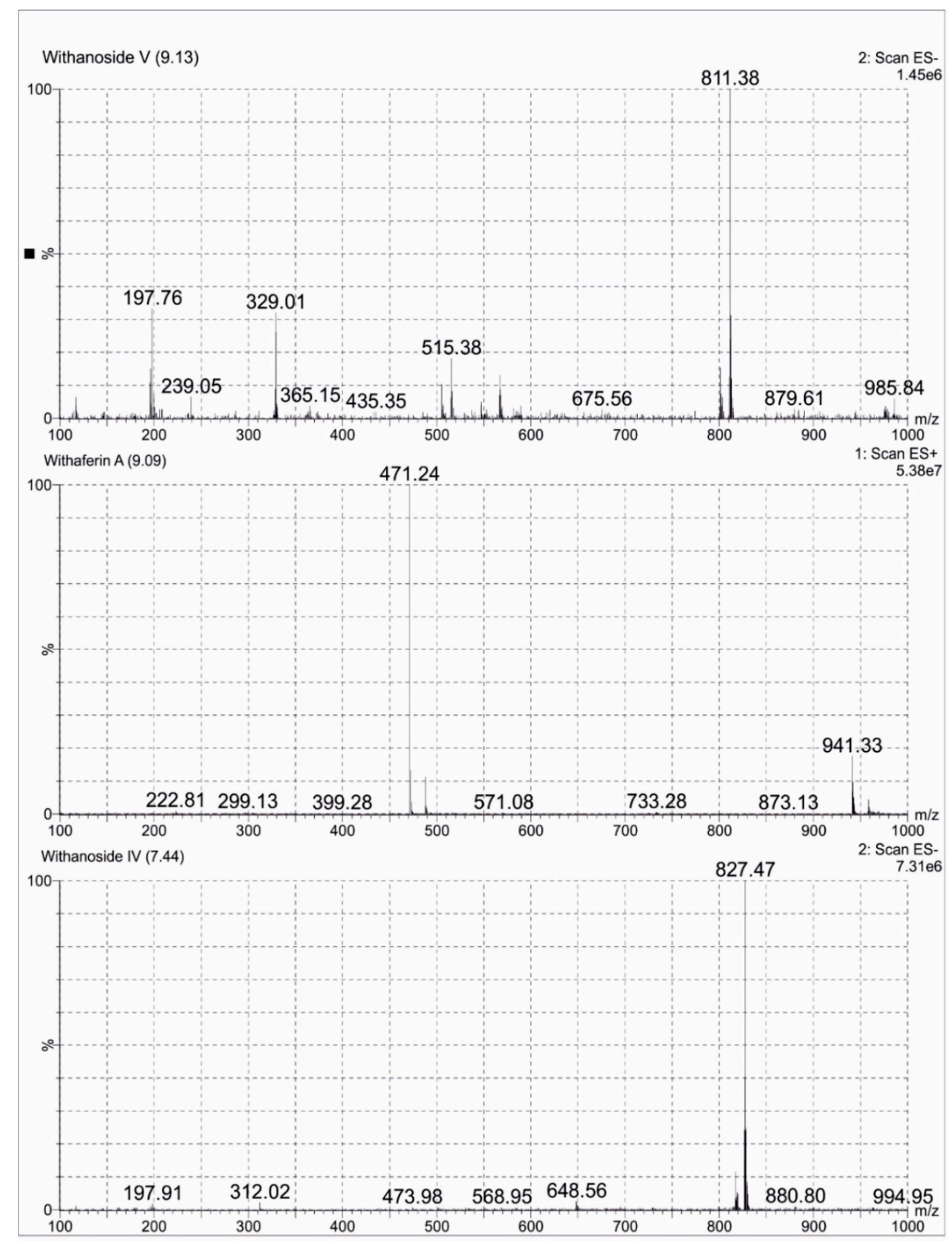

Figure 1. Negative and Positive ion electrospray ionisation liquid chromatography -mass spectrometry mass spectrum (ESI-MS) of Withanoside V, Withaferin A and Withanoside IV separated in Ashwagandha extract. 


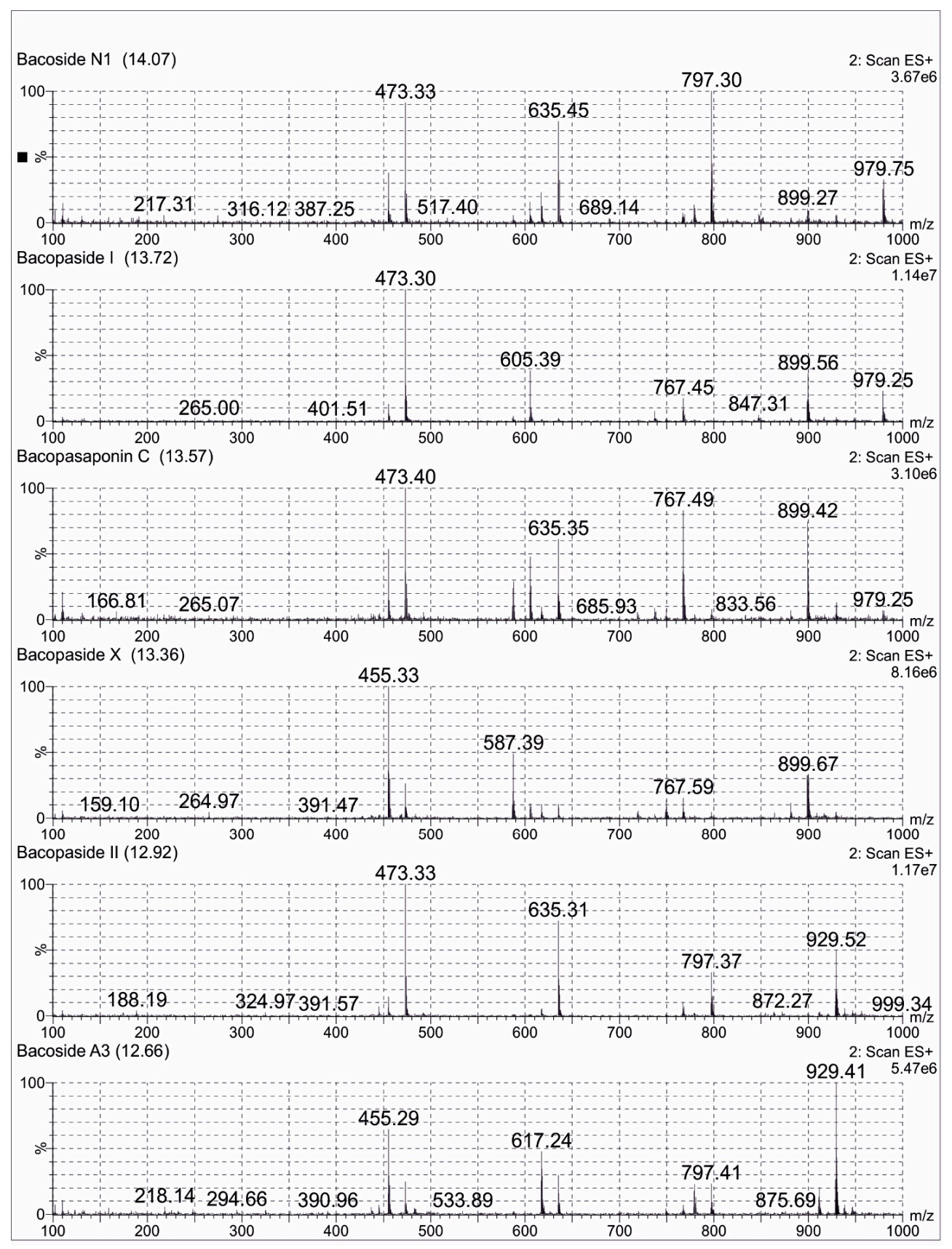

Figure 2. Positive ion electrospray ionisation liquid chromatography -mass spectrometry mass Scheme 1. Bacopaside 1, Bacopasaponin C, Bacopaside X, Bacopaside II and Bacoside A3 separated in Brahmi extract.

\subsection{Animals and Treatment}

Thirty Sprague Dawley rats of 6 to 8 months old, weighing 200-300 g of either sex was used for the study. During the study period, each rat was caged individually in a quiet room under ideal temperature and light. Their normal diet was replaced with a modified liquid diet (MLD) ad libitum [20]. This animal experimental study was conducted in the Department of Pharmacology, Maulana Azad Medical College, Delhi with the approval of the Institutional Animal Ethical Committee of Maulana Azad Medical College. The study was conducted within the framework of CPCSEA (Committee for purpose of control and supervision of experiments on animals) guidelines.

Rats were divided randomly into 6 groups with five animals in each group as follows: Group 1-Vehicle control group treated with Modified liquid diet (MLD) (Vehicle control 
group), Group 2-Modified Liquid diet (MLD) + Ethanol, Group 3-MLD + Ethanol + Diazepam (1 mg/kg IP), Group 4-MLD + Ethanol + Ashwagandha (3.75 mg of withanolide glycosides per $\mathrm{kg}$ body weight), Group 5-MLD + Ethanol + Brahmi (10 mg of bacosides per kg body weight), Group 6-MLD + Ethanol + Ashwagandha + Brahmi ((3.75 mg of withanolide glycosides $+10 \mathrm{mg}$ of bacosides per $\mathrm{kg}$ body weight).

\subsection{Establishment of Alcohol Dependence}

For chronic ethanol exposure, the rats were housed individually and ethanol was given in the modified liquid diet. No extra chow or water was supplied. The composition of the modified liquid diet with ethanol was cow milk $925 \mathrm{~mL}, 25-75 \mathrm{~mL}$ ethanol $(96.5 \%$ ethyl alcohol), vitamin A $5000 \mathrm{IU}$ and sucrose $17 \mathrm{~g}$ [20]. This mixture supplies $1000.7 \mathrm{kcal} / \mathrm{L}$.

At the beginning of the study, all the rats were given the modified liquid diet without ethanol for 7 days. Then, a liquid diet with $2.4 \% v / v$ ethanol was administered for 3 days. The ethanol concentration was increased to $4.8 \% v / v$ for the following 4 days and finally, to $7.2 \% v / v$ for 14 days. A liquid diet was freshly prepared daily and presented at the same time of the day. The weight of the rats was recorded every day. Control rats were fed with an isocaloric liquid diet containing sucrose as a caloric substitute to ethanol. At the end of exposure to $7.2 \% v / v$ ethanol-containing diet, ethanol was withdrawn from the diet after 21 days of administration.

Diazepam (1 mg/ $\mathrm{kg}$ body weight, I.P.), Ashwagandha (3.75 mg of withanolide glycosides per kg body weight, oral), Brahmi (10 mg of bacosides per $\mathrm{kg}$ bodyweight, oral) and Ashwagandha + Brahmi $(3.75 \mathrm{mg}$ of withanolide glycosides $+10 \mathrm{mg}$ of bacosides per $\mathrm{kg}$ body weight) were administered to the rats $45 \mathrm{~min}$ before ethanol withdrawal in the respective groups. Diazepam, Ashwagandha, Brahmi and Ashwagandha + Brahmi were continued once daily dose for 5 days.

\subsection{Assessment}

Behavioural changes, anxiety, locomotor activity and Pentylenetetrazole (PTZ) kindling seizure were assessed by the investigators who were blinded regarding the treatment given to the animals. The rats were observed for $5 \mathrm{~min}$ at second and sixth hours of the withdrawal period for agitation and stereotyped behaviour. After $2 \mathrm{~h}$ and $6 \mathrm{~h}$ of ethanol withdrawal, anxiety was assessed with an elevated plus maze (EPZ) and locomotor activity was assessed with an open field test (OFT). After $6 \mathrm{~h}$ of ethanol withdrawal, the effect of subconvulsive dose of PTZ was assessed.

\subsubsection{Agitation and Stereotyped Behaviour}

Agitation is the state of irritability and aggressive behaviour. Grooming, sniffing, head weaving, gnawing and chewing were observed as major stereotyped behaviours during the ethanol withdrawal in the study. The behaviour of rats was recorded in a video camera and video recordings were reviewed for scoring the behaviour. It was graded as represented in Table $1[23,24]$.

Table 1. Scoring of agitation and stereotyped behaviour.

\begin{tabular}{ccc}
\hline Signs & Scoring & Characteristics Observed \\
\hline \multirow{3}{*}{ Agitation } & 0 & No irritability or aggressive behaviour \\
& 1 & Rats showing mild or moderate irritability \\
& 2 & Very irritable \\
& 3 & handling vocalization and moderately aggressive \\
handling vocalization and very aggressive \\
Stereotyped behaviour & 5 & spontaneous vocalization and very aggressive \\
& 0 & nots shoreotyped behaviour \\
& 2 & two stereotyped behaviour \\
& 3 & three stereotyped behaviour \\
& 4 & four stereotyped behaviour \\
& 5 & all stereotyped behaviour \\
\hline
\end{tabular}




\subsubsection{Elevated Plus Maze}

The elevated plus maze (EPZ) is a plus-shaped apparatus consisting of two opposing closed arms $(50 \mathrm{~cm} \times 10 \mathrm{~cm} \times 40 \mathrm{~cm})$, two opposing open arms $(50 \mathrm{~cm} \times 10 \mathrm{~cm})$ and a central open area, elevated $50 \mathrm{~cm}$ above the ground. An individual rat from each group was placed in the central open area, facing one of the open arms at the beginning of the test. It could freely explore the maze for the duration of $5 \mathrm{~min}$. Anxiolytics were expected to increase the proportion of entries into and time spent in open arms [25].

The whole experiment was set up in dim light conditions and without any human disturbance. Rats were pretreated with vehicle in the control group and test drugs in the drug treated groups orally $45 \mathrm{~min}$ before alcohol withdrawal. The test was performed at $2 \mathrm{~h}$ and $6 \mathrm{~h}$ post withdrawal period. Rats from each group were placed in central area of maze. They were left in the instrument for $5 \mathrm{~min}$. Number of entries and time spent in each arm was recorded for $5 \mathrm{~min}$. The behaviour of each rat was recorded in a video camera. The whole apparatus was wiped with $70 \%$ ethanol before placing the next rat in the maze. Video recording of each rat was later be analysed for the preference of the animal for the first entry, the number of entries into the open and closed arms.

\subsubsection{Open Field Test}

Rats pre-treated orally with vehicle and test drugs 45 min before alcohol withdrawal were tested for locomotor activity in Open field apparatus. At $2 \mathrm{~h}$ and $6 \mathrm{~h}$ post withdrawal period, rats were placed in open field apparatus and were observed for $5 \mathrm{~min}$. The dimensions of the box are 14.2 inch length and breadth which was divided equally into 4 squares. An increase in central locomotion or number of crossings of the lines marked was interpreted as an anxiolytic-like effect while the contrary, that is, a decrease of these variables, was associated with anxiogenic effects [26]. Number of lines crossed by rats in various treatment groups during this period were recorded.

\subsubsection{Pentylenetetrazol Kindling}

Pentylenetetrazole (PTZ) is GABA and Glycine antagonist which is being used to induce seizures in rats. Alcohol withdrawal may lead to convulsions or make animal predisposed to convulsion. Rats entering into convulsions showed convulsive waves axially through the body, Myoclonic jerks and rearing, Clonic forelimb convulsions, Generalized tonic-clonic seizure [25]. Six hours after ethanol withdrawal, seizure threshold was measured in all the groups. Rats were placed under bell jar after administering them with PTZ with a subconvulsive dose of $30 \mathrm{mg} / \mathrm{kg}$ intraperitoneally. The behaviour of rats was observed for $30 \mathrm{~min}$ for onset and duration of convulsions. Number of rats which showed convulsions and percentage of rats convulsed were calculated.

\subsection{Statistical Analysis}

All raw data obtained were preprocessed to check the normal distribution and assumptions of Analysis of Variance prior the analysis. All statistical analysis was done using $R$ version 4.0.2. Variability in the agitation score, stereotypic behaviour, open arm entries and lines crossed for the two time periods were compared using One-way ANOVA with treatment groups as the factor, followed with Tukey's pos-hoc analysis with 95\% level of significance. Weight change in the test subjects between different weeks of observations were derived and compared using a one-way ANOVA with treatment groups as factor, followed with Tukey's post-hoc analysis at 95\% level of significance. A cross-tabulation study with chi-square test at $95 \%$ level of significance was conducted to compare the frequency of convulsion recorded among the test subjects under different treatment groups.

\section{Results}

Agitation score, stereotypic behaviour score, elevated plus maze, open field test and PTZ kindling were observed and compared between the treatment groups and nontreatment group at 2 nd and 6 th hours of alcohol withdrawal. 


\subsection{Body Weight}

It was observed that the change in body weight in all the groups was marginal. There was no significant change in the body weight of each rat which ensured that the modified liquid diet was well substituted their normal feed and ethanol consumption were uniform.

\subsection{Behavioral Changes during Ethanol Withdrawal}

\subsubsection{Agitation Score}

Higher agitation score in MLD + Ethanol group was observed at 2nd hour and 6th hour of alcohol withdrawal. The increase in agitation score when compared with MLD group was statistically significant at 2 nd and 6th hours of alcohol withdrawal $(p<0.001)$. When compared with MLD + Ethanol group, low agitation scores observed in diazepam were statistically significant both at 2 nd and 6th hours $(p<0.001)$. The lower agitation score in Ashwagandha group, when compared with MLD + Ethanol group, was statistically significant at 2nd hour $(p=0.006)$ while it was not statistically significant at 6 th hour. Similarly, mean agitation score in Brahmi treated group were low at 2nd and 6th hours of alcohol withdrawal and when compared to MLD + Ethanol group, the low agitation score was statistically significant at 2nd hour $(p=0.046)$ but not at 6 th hour. In the group administered with Ashwagandha + Brahmi, mean agitation score was significantly low $(p=0.002)$ at 2 nd hour and 6th hour $(p=0.025)$ when compared with MLD + Ethanol (Figure 3A).

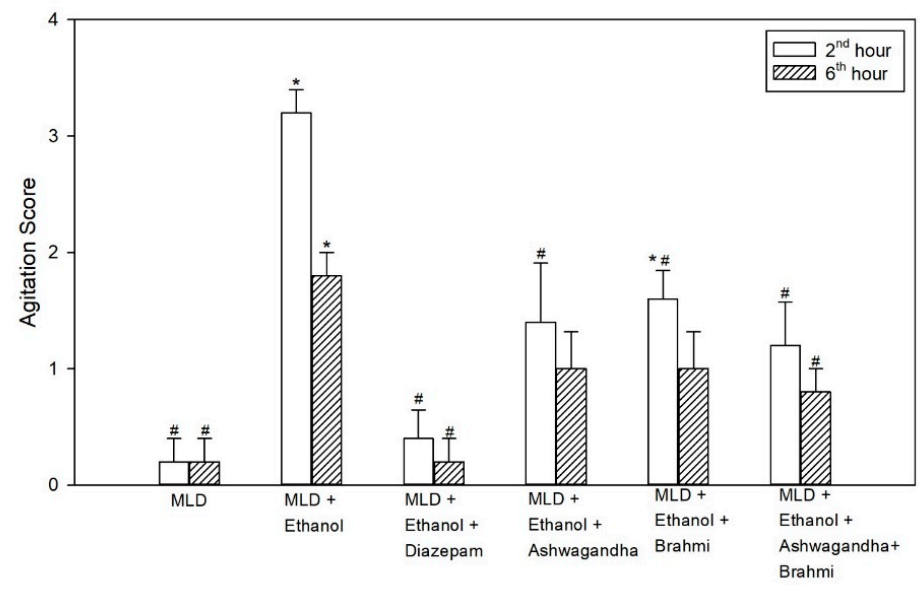

(A)

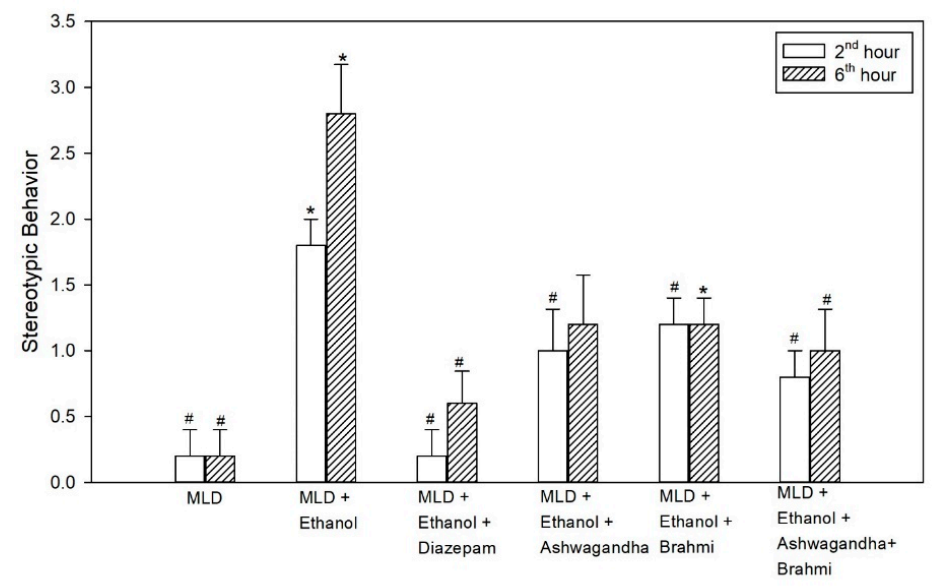

(B)

Figure 3. Effect of test drugs on behavioural changes (A) Agitation (B) Stereotypic behaviour at 2nd hour and 6th hour of alcohol withdrawal in rats. ( ${ }^{(* \prime}$ represents statistically significant compared with MLD group. '\#' represents statistically significance compared with MLD + Ethanol group.). 


\subsubsection{Stereotypic Behaviour}

The higher stereotypic behaviour score observed in the untreated ethanol group when compared with MLD group was statistically significant at 2nd and 6th hours of alcohol withdrawal $(p<0.001)$. Mean stereotypic behaviour score in diazepam treated group when compared with MLD + Ethanol group significantly reduced both at 2nd and 6th hours of alcohol withdrawal $(p<0.001)$. Reduced stereotypic behaviour score in Ashwagandha group, when compared with MLD + Ethanol group, was statistically significant at 2nd hour $(p=0.009)$ while it was not statistically significant at 6 th hour. Similarly, mean stereotypic behaviour score in Brahmi treated group when compared to MLD + Ethanol group was significantly lower at 2 nd hour $(p=0.009)$ and not at 6 th hour. In the group administered with Ashwagandha + Brahmi stereotypic behaviour score was significantly low when compared with MLD + Ethanol group at 2nd hour $(p=0.003)$ and 6th hour $(p=0.043)$ (Figure 3B). Representative video recording of control rat is provided as Supplementary Material (Video S1 Grooming).

\subsection{Elevated Plus Maze (EPZ)}

The decrease in open arm entries in the untreated ethanol group was statistically significant when compared with the MLD group ( $p$ values $=0.001$ and $p=0.009$ ) and in Diazepam treated group, the increase in open arm entries compared with the untreated ethanol group was found to be statistically significant $(p=0.008$ and $p=0.018)$ at 2 nd hour and 6th hour of alcohol withdrawal. However, the increase in open arm entries of the Ashwagandha group was statistically significant at 2 nd hour $(p=0.016)$ and not at 6th hour from the untreated ethanol group. In the group administered with Brahmi, mean open arm entries were not statistically significant from the untreated ethanol group. Similarly, in Ashwagandha + Brahmi treated group, mean open arm entries at 2nd and 6th hour of alcohol withdrawal were statistically significant from the untreated ethanol group ( $p=0.002$ and $p=0.033$ ) (Figure 4). The first entry of rats into an open arm or closed arm of EPZ at 2nd and 6th hour of alcohol withdrawal of all the groups was tabulated in Table 2. Representative video recording of Ashwagandha + Brahmi treated rat is provided as Supplementary Material (Video S2 Elevated Plus Maze).

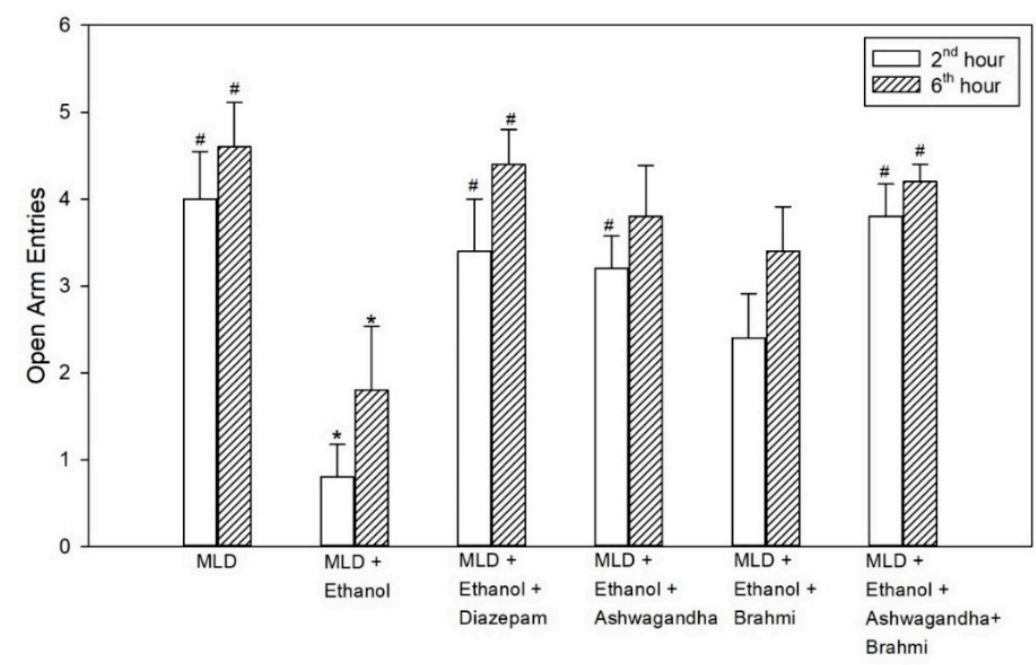

Figure 4. Effect of test drugs on anxiety represented by the number of open arm entries in elevated maze test at 2nd hour and 6th hour of alcohol withdrawal in rats. $\left(^{(* \prime}\right.$ represents statistically significant compared with MLD group. '\#' represents statistically significance compared with MLD + Ethanol group.). 
Table 2. Elevated plus maze first entry in different groups at 2nd and 6th hour of alcohol withdrawal.

\begin{tabular}{ccccc}
\hline \multirow{2}{*}{ Groups } & \multicolumn{3}{c}{ First Entry (Percentage of Rats) } \\
\cline { 2 - 5 } & \multicolumn{3}{c}{$\mathbf{2 ~ h}$} & \multicolumn{2}{c}{$\mathbf{6}$ h } \\
\cline { 2 - 5 } & Open Arm & Closed Arm & Open Arm & Closed Arm \\
\hline MLD & $80 \%$ & $20 \%$ & $80 \%$ & $20 \%$ \\
MLD + Ethanol & $0 \%$ & $100 \%$ & $20 \%$ & $80 \%$ \\
MLD + Ethanol + Diazepam & $60 \%$ & $40 \%$ & $80 \%$ & $20 \%$ \\
MLD + Ethanol + Ashwagandha & $60 \%$ & $40 \%$ & $60 \%$ & $40 \%$ \\
MLD + Ethanol + Brahmi & $40 \%$ & $60 \%$ & $60 \%$ & $40 \%$ \\
MLD + Ethanol + Ashwagandha + Brahmi & $60 \%$ & $40 \%$ & $60 \%$ & $40 \%$ \\
\hline MLD-Modified liquid diet. & & &
\end{tabular}

\subsection{Open Field Test}

The decrease in locomotor activity in the untreated ethanol group compared to MLD group was statistically significant $(p<0.001)$. The increase in locomotor activity observed in the diazepam treated group when compared to the untreated ethanol group was statistically significant both at 2 nd and 6th hour $(p<0.001)$. In the group administered with Ashwagandha, the increase in the number of lines crossed compared to the untreated ethanol group was statistically significant $(<0.001)$. Similarly, in the Brahmi treated group, there was a statistically significant increase in the mean number of lines crossed compared to the untreated ethanol group at 2nd and 6th hour of alcohol withdrawal $(p<0.001)$. In Ashwagandha + Brahmi treated group, mean number of lines crossed in the open field were significantly different from MLD + Ethanol group $(p<0.001)$ (Figure 5). Representative video recording of control rat is provided as Supplementary Material (Video S3 Exploratory Behavior).



Figure 5. Effect of test drugs on locomotor activity represented by the number of lines crossed in open field test at 2nd hour and 6th hour of alcohol withdrawal. (“*' represents statistically significant compared with MLD group. '\#' represents statistically significance compared with MLD + Ethanol group).

\subsection{Pentylenetetrazol (PTZ) Kindling}

In MLD group, administration of sub-convulsive dose of PTZ (30 mg/ $\mathrm{kg}$ i.p) has not shown any convulsions ( $0 \%$ convulsion), whereas the same dose of PTZ administration in MLD + Ethanol group at 6th hour of alcohol withdrawal resulted in the convulsion of all rats (100\% convulsion). In groups treated with diazepam, Ashwagandha, Brahmi and Ashwagandha + Brahmi, no rats developed any convulsion on PTZ administration (30 mg/kg i.p) at 6th hour of alcohol withdrawal ( $0 \%$ convulsion) (Figure 6). Representative 
video recording of MLD + Ethanol treated rat is provided as Supplementary Material (Video S4 PTZ kindling).

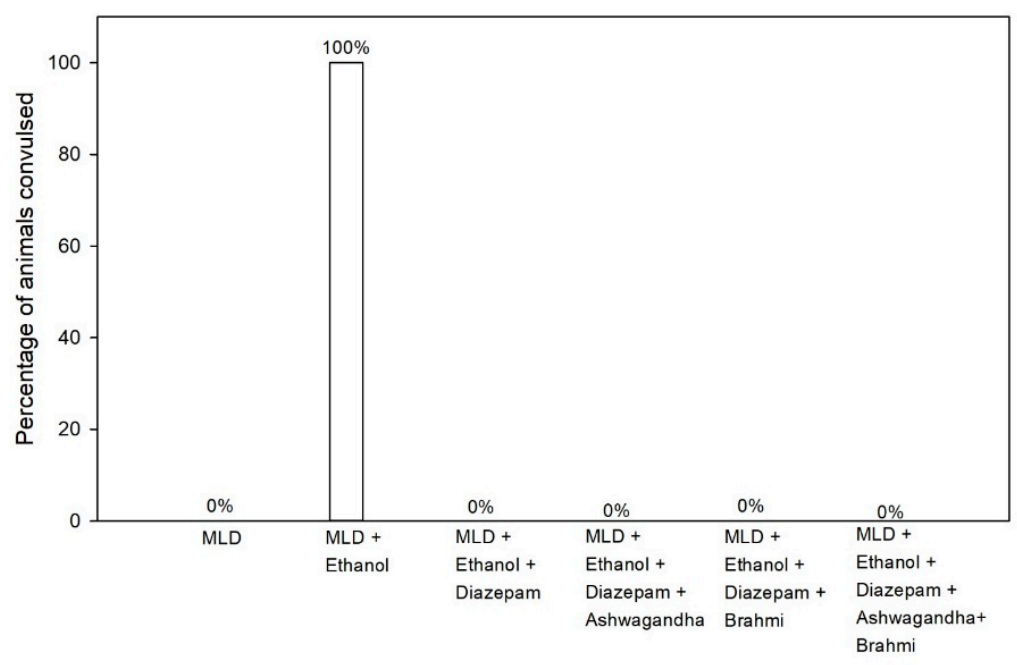

Figure 6. Effect of test drugs on seizures activity represented by the percentage of animals convulsed at 6th hour of alcohol withdrawal after Pentylenetetrazol kindling.

\section{Discussion}

Alcohol abuse is a major health problem in our society. One of the features of chronic alcohol intake is the development of physical dependence, resulting in physical withdrawal reactions after cessation of alcohol. Alcohol withdrawal includes symptoms such as neural excitation (seizures), disorientation, anxiety and agitation [27].

Long-term exposure to alcohol causes adaptive changes in several neurotransmitter systems, especially gamma-aminobutyric acid (GABA) receptors and glutamate receptors. GABA plays a key role in the neurochemical mechanisms on the basis of intoxication, tolerance and withdrawal. Repeated exposure to alcohol reduces GABA hyperpolarization resulting in reduced neural inhibition [28].

Benzodiazepines like diazepam, chlordiazepoxide, etc. are the recommended drugs for the treatment of alcohol withdrawal symptoms because they ameliorate both the anxiety and seizures. They depress CNS excitation by GABA agonism. However, they have potential of causing dependence and leads to abuse. Benzodiazepines also possess serious adverse effects like respiratory failure and delirium [29,30].

Ashwagandha and Brahmi are commonly studied herbs in various CNS disorders including anxiety and seizures [31]. Both of these have shown significant GABA agonist activity in few preclinical studies [16,32]. In our study, we observed an improvement in the behavioural activity of rats with alcohol withdrawal in diazepam, Ashwagandha, Brahmi and Ashwagandha + Brahmi treated groups.

In elevated plus maze (EPM), we observed an anxiolytic effect in alcohol withdrawal rats when treated with Ashwagandha, Brahmi, Ashwagandha + Brahmi and diazepam. The administration of test drugs resulted in first entry of majority of rats into the open arm. In addition, the number of entries into open arms were higher in Ashwagandha, Brahmi and Ashwagandha + Brahmi groups compared with the untreated ethanol group. However, none of these anxiolytic effects were statistically significant when analysed. Previous reports based on the EPM test suggest that withdrawal of ethanol following chronic treatment generates anxiety which is a measure of psychological dependence $[33,34]$. The present studies confirm these by showing a significant reduction in open arm entries in the MLD + Ethanol group at 2nd and 6th hours of alcohol withdrawal. In addition, in a previous study, Ashwagandha showed a significant anxiolytic effect in alcohol withdrawal rats in EPM at 100, 200 and $500 \mathrm{mg} / \mathrm{kg}$ oral dose [10]. 
In the open field test, alcohol withdrawal resulted in reduced locomotor activity at the 2nd and 6th hours. The reduction in exploratory behaviour, often referred to as withdrawal anxiety, was observed by the decrease in the number of line crossing activity. When treated with diazepam, rats showed a significant increase in the number of line crossings. Rats treated with Ashwagandha, Brahmi and Ashwagandha + Brahmi also showed an increase in locomotor activity. In a previous study, Ashwagandha $(500 \mathrm{mg} / \mathrm{kg}$, oral) showed a significant increase in locomotor activity in the open field at 6th hour of alcohol withdrawal [35]. Mishra et al. also reported that rats treated with Ashwagandha ( $3.75 \mathrm{mg}$ of withanolide glycosides per kg body weight) and Brahmi (10 mg of bacosides per kg body weight) were effective in controlling agitation and seizures and showed similar results [36].

Previous studies showed a persistent reduction in the seizure threshold in rats on chronic ethanol administration [35,37]. In this study, Ashwagandha, Brahmi and their combination have suppressed PTZ kindling seizures in alcohol withdrawal rats similar to diazepam. In the previous study, Aswagandha at higher dose $(500 \mathrm{mg} / \mathrm{kg})$ suppressed seizures in a similar manner, whereas Ashwagandha at lower dose $(200 \mathrm{mg} / \mathrm{kg})$ did not suppress seizures at same level [35]. The findings in our study show that both Ashwagandha and Brahmi can have a similar GABA agonist activity comparable to benzodiazepines which offers significant protection against convulsions in alcohol withdrawal syndrome.

\section{Conclusions}

Alcohol withdrawal syndrome is a common manifestation in alcohol dependence due to the imbalance of GABA-NMDA homeostasis in CNS caused by chronic alcohol intake. Similar to diazepam, Ashwagandha and Brahmi individually and in combination significantly reduced the behavioural activity, PTZ kindling seizures and increased the locomotor activity in alcohol withdrawal rats. Further studies at different doses of Ashwagandha and Brahmi can be explored to evaluate their association with alcohol withdrawal syndrome.

Supplementary Materials: The following are available online at https:/ /www.mdpi.com/article/10 .3390/brainsci11070919/s1, Video S1 Grooming; Video S2 Elevated Plus Maze; Video S3 Exploratory Behavior; Video S4 PTZ kindling

Author Contributions: Conceptualization: B.S.K. and S.C.; Data curation: I.M.H. and A.M.; Formal analysis: I.M.H.; Investigation: A.M.; Methodology: I.M.H.; Project administration: A.M.; Supervision: S.C.; Writing—original draft: I.M.H.; Writing—review and editing: B.S.K. and S.C. All authors have read and agreed to the published version of the manuscript.

Funding: This research received no external funding.

Institutional Review Board Statement: The study was conducted according to CPCSEA (Committee for purpose of control and supervision of experiments on animals) guidelines and approved by Institutional Animal Ethical Committee of Maulana Azad Medical College, New Delhi. (Protocol code $01 / 2018$ and date of approval 10 April 2018).

Informed Consent Statement: Not applicable.

Data Availability Statement: The datasets used and/or analysed during the current study are available from the corresponding author on reasonable request.

Acknowledgments: The authors acknowledge Arjuna Natural Private Ltd., India, for providing the extracts of Ashwagandha (Shoden) and Brahmi and their analytical data.

Conflicts of Interest: The authors declare no conflict of interest.

\section{References}

1. Modesto-Lowe, V.; Barron, G.C.; Aronow, B.; Chaplin, M. Gabapentin for alcohol use disorder: A good option, or cause for concern? Cleve Clin. J. Med. 2019, 86, 815. [CrossRef]

2. Perry, E.C. Inpatient management of acute alcohol withdrawal syndrome. CNS Drugs 2014, 28, 401-410. [CrossRef]

3. Hughes, J.R. Alcohol withdrawal seizures. Epilepsy Behav. 2009, 15, 92-97. [CrossRef] 
4. Jesse, S.; Bråthen, G.; Ferrara, M.; Keindl, M.; Ben-Menachem, E.; Tanasescu, R.; Brodtkorb, E.; Hillbom, M.; Leone, M.A.; Ludolph, A.C. Alcohol withdrawal syndrome: Mechanisms, manifestations, and management. Acta Neurol. Scand. 2017, 135, 4-16. [CrossRef]

5. Long, D.; Long, B.; Koyfman, A. The emergency medicine management of severe alcohol withdrawal. Am. J. Emerg. Med. 2017, 35, 1005-1011. [CrossRef] [PubMed]

6. Airagnes, G.; Ducoutumany, G.; Laffy-Beaufils, B.; Le Faou, A.-L.; Limosin, F. Alcohol withdrawal syndrome management: Is there anything new? Rev. Med. Interne 2019, 40, 373-379. [CrossRef] [PubMed]

7. Winters, M. Ancient medicine, modern use: Withania somnifera and its potential role in integrative oncology. Altern. Med. Rev. 2006, 11, 269-277. [PubMed]

8. Schachter, S.C. Botanicals and herbs: A traditional approach to treating epilepsy. Neurotherapeutics 2009, 6, 415-420. [CrossRef] [PubMed]

9. Gomes, N.G.M.; Campos, M.G.; Órfão, J.M.C.; Ribeiro, C.A.F. Plants with neurobiological activity as potential targets for drug discovery. Prog. Neuro-Psychopharmacol. Biol. Psychiatry 2009, 33, 1372-1389. [CrossRef] [PubMed]

10. Gupta, G.L.; Rana, A.C. Effect of withania somnifera dunal in ethanol-induced anxiolysis and withdrawal anxiety in rats. Indian J. Exp. Biol. 2008, 46, 470-475.

11. Sreedevi, C.D.; Latha, P.G.; Ancy, P.; Suja, S.R.; Shyamal, S.; Shine, V.J.; Sini, S.; Anuja, G.I.; Rajasekharan, S. Hepatoprotective studies on Sida acuta Burm. J. Ethnopharmacol. 2009, 124, 171-175. [CrossRef]

12. Bhattacharya, S.K.; Kumar, A.; Ghosal, S. Effects of glycowithanolides from Withania somnifera on an animal model of Alzheimer's disease and perturbed central cholinergic markers of cognition in rats. Phytother. Res. 1995, 9, 110-113. [CrossRef]

13. Kuboyama, T.; Tohda, C.; Komatsu, K. Withanoside IV and its active metabolite, sominone, attenuate A $\beta$ (25-35)-induced neurodegeneration. Eur. J. Neurosci. 2006, 23, 1417-1426. [CrossRef]

14. Kulkarni, S.K.; Dhir, A. Withania somnifera: An Indian ginseng. Prog. Neuro-Psychopharmacol. Biol. Psychiatry 2008, 32, 1093-1105. [CrossRef] [PubMed]

15. Sumathi, T.; Nongbri, A. Hepatoprotective effect of Bacoside-A, a major constituent of Bacopa monniera Linn. Phytomedicine 2008, 15, 901-905. [CrossRef] [PubMed]

16. Mathew, J.; Balakrishnan, S.; Antony, S.; Abraham, P.M.; Paulose, C.S. Decreased GABA receptor in the cerebral cortex of epileptic rats: Effect of Bacopa monnieri and Bacoside-A. J. Biomed. Sci. 2012, 19, 1-13. [CrossRef]

17. Vollala, V.R.; Upadhya, S.; Nayak, S. Effect of Bacopa monniera Linn.(brahmi) extract on learning and memory in rats: A behavioral study. J. Vet. Behav. 2010, 5, 69-74. [CrossRef]

18. Mannan, A.; Abir, A.B.; Rahman, R. Antidepressant-like effects of methanolic extract of Bacopa monniera in mice. BMC Complement. Altern. Med. 2015, 15, 337. [CrossRef] [PubMed]

19. Gong, M.-F.; Wen, R.-T.; Xu, Y.; Pan, J.-C.; Fei, N.; Zhou, Y.-M.; Xu, J.-P.; Liang, J.-H.; Zhang, H.-T. Attenuation of ethanol abstinenceinduced anxiety-and depressive-like behavior by the phosphodiesterase-4 inhibitor rolipram in rodents. Psychopharmacology 2017, 234, 3143-3151. [CrossRef]

20. Uzbay, I.T.; Kayaalp, S.O. A modified liquid diet of chronic ethanol administration: Validation by ethanol withdrawal syndrome in rats. Pharmacol. Res. 1995, 31, 37-42. [CrossRef]

21. Girme, A.; Saste, G.; Pawar, S.; Balasubramaniam, A.K.; Musande, K.; Darji, B.; Satti, N.K.; Verma, M.K.; Anand, R.; Singh, R.; et al. Investigating 11 Withanosides and Withanolides by UHPLC-PDA and Mass Fragmentation Studies from Ashwagandha (Withania somnifera). ACS Omega 2020, 5, 27933-27943. [CrossRef]

22. Nuengchamnong, N.; Sookying, S.; Ingkaninan, K. LC-ESI-QTOF-MS based screening and identification of isomeric jujubogenin and pseudojujubogenin aglycones in Bacopa monnieri extract. J. Pharm. Biomed. Anal. 2016, 129, 121-134. [CrossRef]

23. Sağlam, E.; Kayir, H.; Çelik, T.; Uzbay, T. Effects of escitalopram on ethanol withdrawal syndrome in rats. Prog. NeuroPsychopharmacol. Biol. Psychiatry 2006, 30, 1027-1032. [CrossRef]

24. Sağlam, E.; Uzbay, I.T.; Kayir, H.; Çelik, T.; Beyazyürek, M. Effects of venlafaxine on ethanol withdrawal syndrome in rats. Fundam. Clin. Pharmacol. 2004, 18, 693-698. [CrossRef]

25. Medhi, B.; Prakash, A. Practical Manual of Experimental and Clinical Pharmacology; Jaypee Brothers Medical Publishers: New Delhi, India, 2010; ISBN 8184489536.

26. Barua, C.C.; Talukdar, A.; Begum, S.A.; Borah, P.; Lahkar, M. Anxiolytic activity of methanol leaf extract of Achyranthes aspera Linn in mice using experimental models of anxiety. Indian J. Pharmacol. 2012, 44, 63. [CrossRef]

27. Jane, L. How is alcohol withdrawal syndrome best managed in the emergency department? Int. Emerg. Nurs. 2010, 18, 89-98. [CrossRef]

28. Leggio, L.; Kenna, G.A.; Swift, R.M. New developments for the pharmacological treatment of alcohol withdrawal syndrome. A focus on non-benzodiazepine GABAergic medications. Prog. Neuro-Psychopharmacol. Biol. Psychiatry 2008, 32, $1106-1117$. [CrossRef]

29. Liang, J.; Olsen, R.W. Alcohol use disorders and current pharmacological therapies: The role of GABA A receptors. Acta Pharmacol. Sin. 2014, 35, 981-993. [CrossRef]

30. Maldonado, J.R. Novel algorithms for the prophylaxis and management of alcohol withdrawal syndromes-beyond benzodiazepines. Crit. Care Clin. 2017, 33, 559-599. [CrossRef] 
31. Mannangatti, P.; Naidu, K.N. Indian herbs for the treatment of neurodegenerative disease. In The Benefits of Natural Products for Neurodegenerative Diseases; Springer: Berlin, Germany, 2016; pp. 323-336.

32. Candelario, M.; Cuellar, E.; Reyes-Ruiz, J.M.; Darabedian, N.; Feimeng, Z.; Miledi, R.; Russo-Neustadt, A.; Limon, A. Direct evidence for GABAergic activity of Withania somnifera on mammalian ionotropic GABAA and GABA $\rho$ receptors. J. Ethnopharmacol. 2015, 171, 264-272. [CrossRef]

33. Kokare, D.M.; Chopde, C.T.; Subhedar, N.K. Participation of $\alpha$-melanocyte stimulating hormone in ethanol-induced anxiolysis and withdrawal anxiety in rats. Neuropharmacology 2006, 51, 536-545. [CrossRef]

34. Gatch, M.B.; Wallis, C.J.; Lal, H. Effects of ritanserin on ethanol withdrawal-Induced anxiety in rats. Alcohol 2000, 21, 11-17. [CrossRef]

35. Ruby, B.; Benson, M.K.; Kumar, E.P.; Sudha, S.; Wilking, J.E. Evaluation of Ashwagandha in alcohol withdrawal syndrome. Asian Pac. J. Trop. Dis. 2012, 2, S856-S860. [CrossRef]

36. Mishra, A.; Gupta, P.; Kalra, B.S.; Tiwari, Y.K. Efficacy of Ashwagandha and Brahmi Extract on Alcohol Withdrawal Syndrome in Laboratory Rats. Int. J. Pharmacol. 2020, 16, 343-350. [CrossRef]

37. Kokka, N.; Sapp, D.W.; Taylor, A.M.; Olsen, R.W. The kindling model of alcohol dependence: Similar persistent reduction in seizure threshold to pentylenetetrazol in animals receiving chronic ethanol or chronic pentylenetetrazol. Alcohol. Clin. Exp. Res. 1993, 17, 525-531. [CrossRef] [PubMed] 